\title{
A system for accurately measuring articulatory durations which is not reset by any included silen periods:
}

\section{KEVIN DONAHOO†, NORMAN NETTLETON and JOHN BRADSHAW \\ Monash University, Clayton, Victoria 3168, Australia}

A device is described that accurately measures duration of phonation or articulation without being reset by intervening silent periods or pauses, which may be within the range of 10 to $1,000 \mathrm{msec}$. Thus, it is possible to time the overall duration of single sounds, syllables, single or multiple words, even though periods of silence are included. Input can be direct or recorded, the ancilliaries being a voice-operated switch and a chronoscope.

The acoustic characteristics of speech sounds are considerably more complex than the sounds normally used in investigating the auditory system. A "continuous" speech signal, if transduced into a visual display on a speech spectrograph (cf. Cooper, 1950; Liberman, Cooper, Shankweiler, \& Studdert-Kennedy, $1967)$, is seen to consist of a number of concentrations of acoustic energy (formants) within certain characteristic frequencies. The important ones range in frequency from a few hundred to nearly $3,000 \mathrm{~Hz}$; their steady-state components characterize particularly the vowels and continuants in speech, while the formant transitions (frequency changes) convey information about stop consonants. The average intensity of the signal may range from the merest whisper to a level perhaps even in excess of $100 \mathrm{~dB}$, and it can be seen from the spectrographic record that within a word, or even within a unit as apparently continuous as the syllable, there may be silent periods as long as $100 \mathrm{msec}$. These silent periods convey phonetic information and may, for example, lead to the perceptual distinction between "slit" and "split" (Huggins. 1972). Frequently, it is desired, both in studying the motor aspects of articulation for their own sake and in measuring time constants in vocalization, to bypass the lengthy and often complex spectrographic record. A simple display, e.g., a storage oscilloscope, can rarely provide a sufficiently accurate measure. When initiation latencies alone are studied, a simple voice key might suffice, as long as its response characteristics. in terms of frequency and intensity, are adequate. In some cases, measures of articulatory duration may be required. for example, when $S$ is subjected to delayed auditory feedback (DAF). If the vocalization has a clearly defined beginning and end, it would seem a simple matter to set

\footnotetext{
* Support was provided by the Australian Research Grants Committee to the last author for a project for which the device was developed.

+Address requests for reprints to firin Donahoo, Department of Psychology, Monash Universits. Clayton. Victoria 3168, Australia.
}

up a system incorporating a Schmitt trigger to signal the onset and offset events. However, the apparently continuous nature of the vocalization may be a perceptual artifact in that pauses or silent periods may be included between the two boundaries. A system designed to cope with such problems, so that not only initiation but duration times could be measured accurately, is described below. This obviates the necessity for either spectrographic or costly and complicated computer analysis. This system is accurate to $1 \mathrm{msec}$, when used with either a computer or conventional chronoscope facilities with $.1-\mathrm{msec}$ resolution, and consists of a voice-operated switch (Stage 1) and a variable delay unit (Stage 2).

\section{APPARATUS \\ Principles of Operation}

The train of pulses from a voice-operated switch (Stage 1) is used to operate two separate circuits in the variable delay unit (Stage 2). One is the start-pulse circuit; the more important is the delayed stop-pulse circuit. The delay is introduced to the stop pulse to override any "troughs," or zero-crossing points, in the frequency content of the word; these "troughs" would normally cause the stop pulse to be delivered prematurely. The delay can also be set long enough to ignore pauses between words if more than one word is to be measured.

\section{Voice-Operated Switch}

Stage 1 (see Fig. 1) is a voice-operated switch consisting of two operational amplifier stages, a comparator and associated relay and lamp-drive circuits. Speech input can be either direct (preferably from a microphone of the cardioid type) or from a tape recorder.

The input circuit has a gain of up to 40.000 , depending on the sensitivity level set, and speech output from this amplifier is in a highly clipped form. The comparator output is simply a squared version of the input in a form acceptable to transistor-transistor logic, and is used to drive a 1 -msec monostable. The monostable pulse output passes through an OR gate with the comparator output, and the resultant train of pulses is fed to the delay unit, which supplies the chronoscope start/stop pulses. Other outputs available from the voice-operated switch include a set of changeover contacts and positive and negative pulses which correspond to the speech onset.

\section{Variable Delay Unit}

Stage ? (see Fig. 2), the variable delay unit, has two 
Table 1

Component List for Stage 1

\begin{tabular}{ll} 
IC 1 & LM 312 \\
IC 2 & LM 741 \\
IC 3 & LM 311 \\
IC 4, IC 5 & 74121 \\
IC 6 & 7400 \\
IC 7 & 7402 \\
R 1 Relay & $5-V 34-$ Ohm Coil \\
Microphone & Leson TW-209 \\
Resistors & All 5\% Tolerance \\
\hline
\end{tabular}

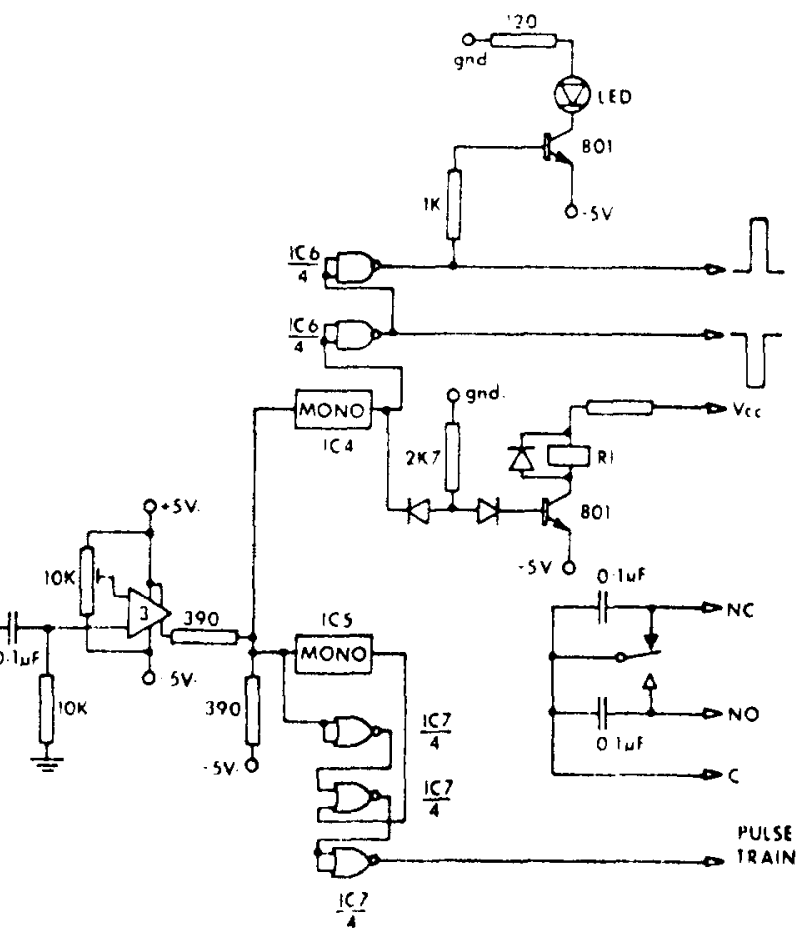

Fig. 1. Circuit diagram for voice-operated switch (Stage 1). For list of components, see Table 1.

separate circuits operated by the train of pulses from the flip-flop. Two stages of a hex inverter ICl are used as an voice-operated switch.

\section{Start Circuit}

The voltage input from the voice-operated switch is used to trigger a change of state in IC2, a SN7474 "D" input buffer and inverter. The flip-flop output drives a monostable multivibrator, which delivers a $2-\mathrm{msec}$ negative transition pulse to start the chronoscope. There is a lamp circuit, also driven by the flip-flop, which is used as a reset/ready indicator. The circuit is placed in

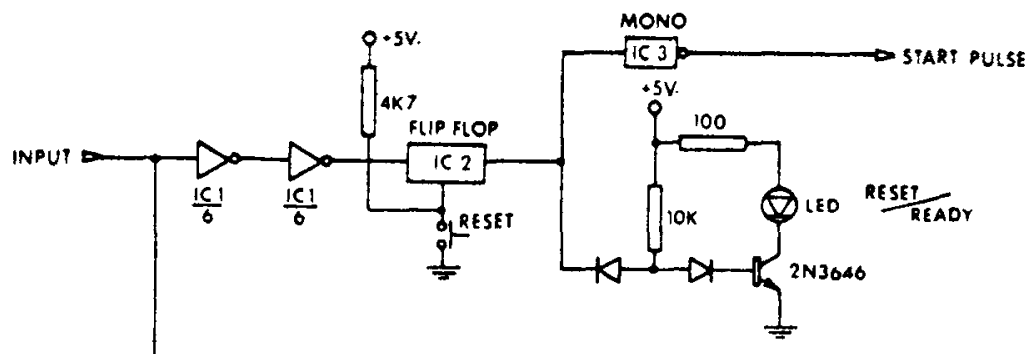

Table 2

Component List for Stage 2

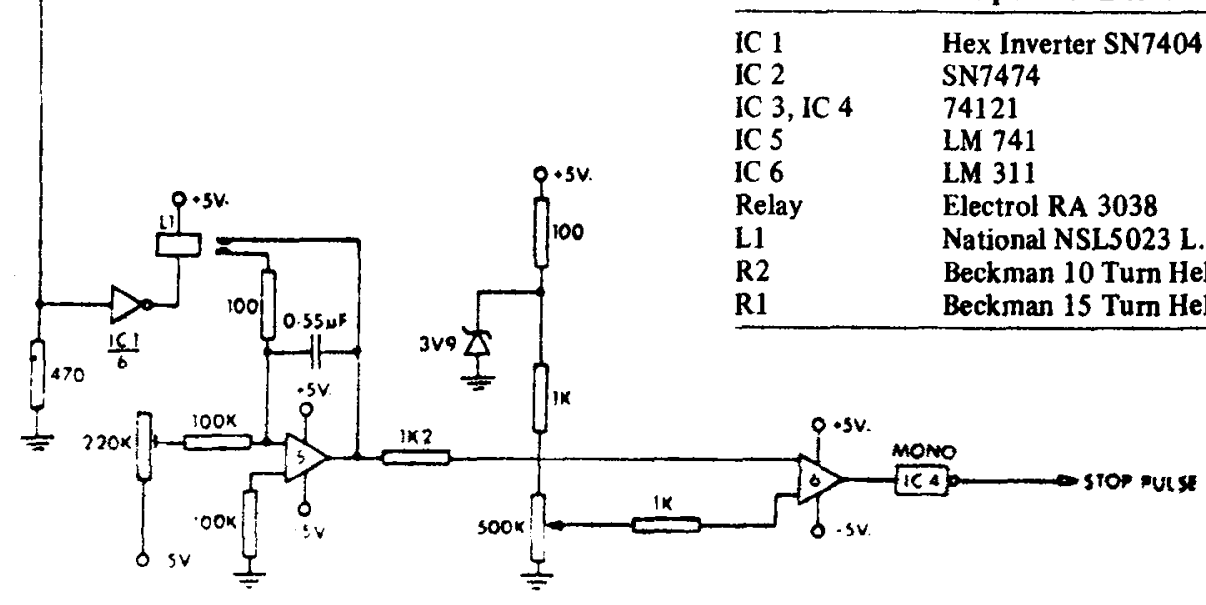

Fig. 2. Circuit diagram for variable delay unit (Stage 2). For list of components, see Table 2. 
reset/ready mode by the operator pushing the reset button, or when a contact closure is made across the reset terminals. The device will not deliver a chronoscope start pulse until it has been reset, thus preventing false triggering from a spurious input to the microphone.

\section{Stop Circuit}

When the train of pulses is triggering the start circuit, the same positive-going input voltage is holding closed a pair of contacts across the integrating capacitor. This is achieved by a $5 \cdot \mathrm{V}$ logic-compatible reed relay driven by buffer (IC1/6) from the input.

As soon as the input disappears, the contacts open and the integrator, IC5, begins to ramp at about $40 \mathrm{~V} / \mathrm{sec}$. RI sets the integrating period. The integrator output is compared to a reference voltage set by R2 (front panel delay control), and as soon as the two are equal, the comparator IC6 changes its output state, and a second monostable multivibrator delivers a 200 -microsec chronoscope stop pulse. This circuit can handle two overlapping delay periods of $10-100 \mathrm{msec}$ and $10-1,000 \mathrm{msec}$. Resolution in the former mode is within 100 microsec; in the latter, it is within $1 \mathrm{msec}$. The method of varying a comparator reference voltage to change delays, rather than alter the integrator ramp time, was introduced to overcome the problems of setting-up and calibration caused by changing the values of $\mathrm{Rl}$ and $\mathrm{Cl}$ in the integrator (see Fig. 2). Virtually any delay time can be established simply by arranging the ramp time to be in excess of the delay required (by a factor of 10,100 , etc., on the original ramp time) and varying the comparator reference voltage as a final adjustment. Thus, while the initial ramp time may be
$120 \mathrm{msec}(40 \mathrm{~V} / \mathrm{sec})$ and delays may range from 10 to $100 \mathrm{msec}$, delays of up to $1,000 \mathrm{msec}$ can be selected by changing ramp time to approximately $1,200 \mathrm{msec}$, i.e., by multiplying by a factor of 10 .

The chronoscope will display a response time equal to the length of the word plus the delay time set. Simple subtraction will produce the word length in milliseconds. Once calibrated, the complete system will stay accurate to within $1 \mathrm{msec}$ if good regulated supplies are used and the integrator components are kept to close tolerance.

\section{CALIBRATION}

All that is required to calibrate the instrument is a function generator capable of supplying variable-duration tone bursts, e.g., the Wavetek 134. A $100-\mathrm{msec}$ tone burst of $500-\mathrm{Hz}$ square wave is fed directly to the variable delay unit (Stage 2). The delay control, R2, is set to maximum of $100 \mathrm{msec}$, and $\mathrm{R} 1$ is adjusted to give a reading of $200 \mathrm{msec}$ on the chronoscope. The circuit is now calibrated, and R2 assumes linear control of delays from 10 to $100 \mathrm{msec}$. The same procedure is used for any extended delay period, i.e., up to $1,000 \mathrm{msec}$.

\section{REFERENCES}

Cooper, F. S. Spectrum analysis. Journal of the Acoustical Society of America, 1950, 22, 761-762.

Huggins, A. W. F. On the perception of temporal phenomena in speech. Journal of the Acoustical Society of America, 1972, $51,1279-1290$.

Liberman, A. M., Cooper, F. S., Shankweiler, D., \& Studdert-Kennedy, M. Perception of the speech code. Psychological Review, 1967, 74, 431-461.

(Received for publication May 12, 1973; accepted June 4, 1973.) 